Algebraic 85 Geometric Topology

Volume 3 (2003) 569-586

Published: 20 June 2003

ATG

\title{
Open books and configurations of symplectic surfaces
}

\author{
DAVID T. GAY
}

\begin{abstract}
We study neighborhoods of configurations of symplectic surfaces in symplectic 4-manifolds. We show that suitably "positive" configurations have neighborhoods with concave boundaries and we explicitly describe open book decompositions of the boundaries supporting the associated negative contact structures. This is used to prove symplectic nonfillability for certain contact 3-manifolds and thus nonpositivity for certain mapping classes on surfaces with boundary. Similarly, we show that certain pairs of contact 3-manifolds cannot appear as the disconnected convex boundary of any connected symplectic 4-manifold. Our result also has the potential to produce obstructions to embedding specific symplectic configurations in closed symplectic 4-manifolds and to generate new symplectic surgeries. From a purely topological perspective, the techniques in this paper show how to construct a natural open book decomposition on the boundary of any plumbed 4-manifold.
\end{abstract}

AMS Classification 57R17; 57N10, 57N13

Keywords Symplectic, contact, concave, open book, plumbing, fillable

\section{Main Results}

All manifolds in this paper are oriented; if $(X, \omega)$ is a symplectic 4-manifold we assume that $\omega \wedge \omega>0$. By a symplectic configuration in a symplectic 4-manifold $(X, \omega)$, we mean a union $C=\Sigma_{1} \cup \ldots \cup \Sigma_{n}$ of closed symplectic surfaces embedded in $(X, \omega)$ such that all intersections between surfaces are $\omega$-orthogonal.

A symplectic configuration graph is a labelled graph $G$ with no edges from a vertex to itself and with each vertex $v_{i}$ labelled with a triple $\left(g_{i}, m_{i}, a_{i}\right)$, where $g_{i} \in\{0,1,2, \ldots\}, m_{i} \in \mathbb{Z}$ and $a_{i} \in(0, \infty)$. Associated to a symplectic configuration $C=\Sigma_{1} \cup \ldots \cup \Sigma_{n}$ in a symplectic 4 -manifold $(X, \omega)$ is a symplectic configuration graph $G(C)$ where each vertex $v_{i}$ corresponds to a surface $\Sigma_{i}$, 
$g_{i}=\operatorname{genus}\left(\Sigma_{i}\right), m_{i}=\Sigma_{i} \cdot \Sigma_{i}$ and $a_{i}=\int_{\Sigma_{i}} \omega$, and where each edge represents a point of intersection. Because $\omega$-orthogonal intersections are necessarily positive, $G(C)$ completely determines the topology of a regular neighborhood of $C$ (namely, the result of plumbing disk bundles over surfaces according to $G(C)$ ); for this much the areas $\left\{a_{i}\right\}$ are irrelevant. If we include the area information then $G(C)$ also determines the germ of $\omega$ near $C$ (due to a slight generalization of standard symplectic neighborhood theorems, as in [10]).

For any vertex $v_{i}$ in a graph $G$, let $d_{i}$ denote the degree of $v_{i}$, the number of edges connected to $v_{i}$. We say that a configuration graph $G$ is positive if $m_{i}+d_{i}>0$ for every vertex $v_{i}$.

Recall that the boundary of a symplectic 4-manifold $(X, \omega)$ is concave (resp. convex) if there exists a symplectic dilation $V$ defined on a neighborhood of $\partial X$ pointing in (resp. out) along $\partial X$; this induces a negative (resp. positive) contact structure $\xi=\left.\operatorname{ker} \imath_{V} \omega\right|_{\partial X}$ on $\partial X$.

We present our main theorem in two parts. Part A states that positive symplectic configurations have neighborhoods with concave boundaries, and part B explicitly describes the contact structures on such boundaries in terms of open book decompositions.

Theorem 1.1 (part A) Given any positive symplectic configuration graph $G$ there exists an open symplectic 4-manifold $(N(G), \omega(G))$, a symplectic configuration $C(G) \subset(N(G), \omega(G))$ and a positive contact 3-manifold $(M(G), \xi(G))$, satisfying the following properties:

- $G=G(C(G))$.

- For some contact form $\alpha$ for $\xi(G)$ and some (not necessarily smooth) function $f: M(G) \rightarrow \mathbb{R}$, letting $X_{f}=\{(t, p) \mid t<f(p)\} \subset \mathbb{R} \times M(G)$ and $\omega=d\left(e^{t} \alpha\right)$, there is a symplectomorphism $\phi:\left(X_{f}, \omega\right) \rightarrow(N(G) \backslash$ $C(G), \omega(G))$ such that

$$
C(G)=\left\{\lim _{t \rightarrow f(p)} \phi(t, p) \mid p \in M(G)\right\} .
$$

Thus, given any symplectic configuration $C$ in any symplectic 4-manifold $(X, \omega)$, if $G(C)=G$ then there exists a compact neighborhood of $C$ in $(X, \omega)$ which is symplectomorphic to a neighborhood of $C(G)$ in $(N(G), \omega(G))$ and which has concave boundary contactomorphic to $(-M(G), \xi(G))$.

The strength of this theorem will lie in the characterization of $(M(G), \xi(G))$ in terms of an open book decomposition of $M(G)$. We briefly recall the relationship between contact structures and open books; for more details see [5] and [3]. 
An open book decomposition of a 3 -manifold $M$ is a pair $(L, p)$, where $L$ is a link and $p: M \backslash L \rightarrow S^{1}$ is a fibration such that the fibers are longitudinal near each component of $L$. The link $L$ is called the binding and the compact surfaces $\Sigma_{t}=p^{-1}(t) \cup L$ are called the pages, with $L=\partial \Sigma_{t}$ for all $t \in S^{1}$. By the mapping class group $\mathcal{M}(\Sigma)$ for a compact surface $\Sigma$ with boundary, we mean the group of orientation-preserving self-diffeomorphisms of $\Sigma$ fixing $\partial \Sigma$ pointwise modulo isotopies fixing $\partial \Sigma$ pointwise. The monodromy of an open book is the mapping class $h \in \mathcal{M}\left(\Sigma_{0}\right)$ given by the return map of a flow transverse to the pages and meridinal near the binding.

A positive contact form $\alpha$ on $M$ is supported by the open book $(L, p)$ if $d \alpha$ is positive on each page and if $\alpha$ orients $L$ in the same sense that $L$ is oriented as the boundary of a page. A positive contact structure $\xi$ is supported by $(L, p)$ if $\xi=\operatorname{ker} \alpha$ for some contact form $\alpha$ which is supported by $(L, p)$. We have the following result at our disposal:

Theorem 1.2 (Thurston-Winkelnkemper [12], Torisu [13], Giroux [5]) Every open book decomposition of any 3-manifold supports some positive contact structure, and any two positive contact structures supported by the same open book are isotopic.

Thus, given a compact surface $\Sigma$ with boundary and a mapping class $h \in$ $\mathcal{M}(\Sigma)$, there exists a unique (up to contactomorphism) positive contact 3manifold with contact structure supported by an open book with page $\Sigma$ and monodromy $h$; we denote this contact manifold $\mathcal{B}(\Sigma, h)$.

Given a positive configuration graph $G$, for each vertex $v_{i}$ let $F_{i}$ be a surface of genus $g_{i}$ with $m_{i}+d_{i}$ boundary components. Let $\Sigma(G)$ be the surface resulting from performing connect sums between these surfaces, with one connect sum between $F_{i}$ and $F_{j}$ for each edge connecting $v_{i}$ to $v_{j}$. Each edge in $G$ corresponds to a circle in $\Sigma(G)$. An example of a graph $G$ and the surface $\Sigma(G)$ is illustrated in figure 1 , with the circles corresponding to the edges drawn in dashed lines. Let $\sigma(G)$ be the product of one right-handed Dehn twist around each of the circles in $\Sigma(G)$ corresponding to the edges of $G$, let $\delta(G)$ be the product of one right-handed Dehn twist around each component of $\partial \Sigma(G)$ and let $h(G)=\sigma(G)^{-1} \circ \delta(G)$.

Theorem 1.1 (part B) $(M(G), \xi(G))=\mathcal{B}(\Sigma(G), h(G))$

Note that the area information recorded in the graph $G$ is not recorded on the boundary $(M(G), \xi(G))$. For this reason, we may suppress mention of the areas 

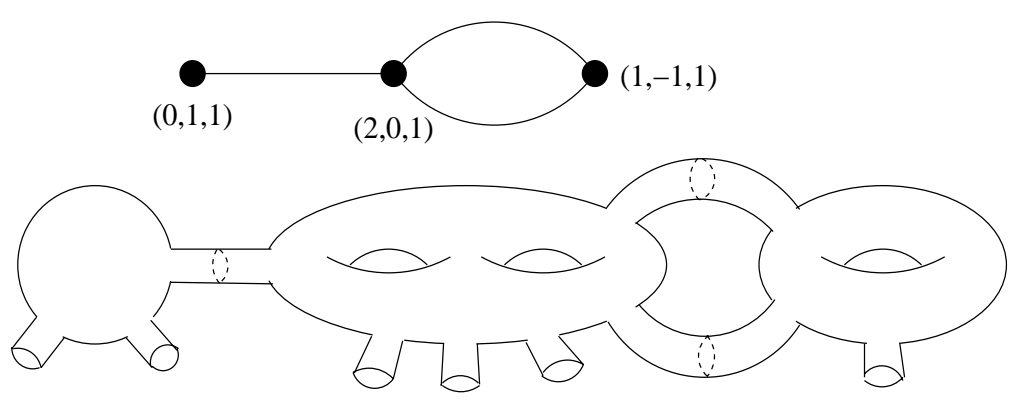

Figure 1: A symplectic configuration graph $G$ and the surface $\Sigma(G)$

and think of the vertices of $G$ as labelled with pairs $(g, m)$, rather than triples $(g, m, a)$.

After proving theorem 1.1 we will briefly discuss the situation when $G$ is not positive.

Theorem 1.1 also has purely topological content, namely the explicit characterization of an open book on the boundary of a plumbed 4-manifold corresponding to a positive configuration graph. If we are only interested in smooth topology, the techniques used to prove theorem 1.1 do yield a theorem characterizing open books on the boundaries of arbitrary plumbed 4-manifolds, which we now state.

Let a plumbing graph be a graph $G$ with no edges connecting a vertex to itself, with each vertex $v_{i}$ labelled with a pair $\left(g_{i}, m_{i}\right)$ and with each edge labelled with $\mathrm{a}+$ or $\mathrm{a}-$. The plumbed 4 -manifold $X(G)$ corresponding to $G$ is a neighborhood of a configuration of surfaces $\Sigma_{1} \cup \ldots \cup \Sigma_{n}$ corresponding to the vertices $v_{1}, \ldots, v_{n}$ of $G$, with genus $\left(\Sigma_{i}\right)=v_{i}, \Sigma_{i} \cdot \Sigma_{i}=m_{i}$ and each + (resp. -) edge corresponding to a positive (resp. negative) transverse intersection between two surfaces. For each vertex $v_{i}$ let $d_{i}$ be the signed count of edges connecting to $v_{i}(\mathrm{a}+$ edge contributes +1 while a - edge contributes -1$)$. For each $v_{i}$ let $F_{i}$ be a surface of genus $g_{i}$ with $\left|m_{i}+d_{i}\right|$ boundary components and let $\Sigma(G)$ be the result of performing connect sums between these surfaces according to $G$. Let $h(G)$ be the product of the following Dehn twists: one right-handed Dehn twist about each circle in $\Sigma(G)$ corresponding to a + edge in $G$, one left-handed Dehn twist about each circle in $\Sigma(G)$ corresponding to a - edge, one left-handed Dehn twist about each boundary component coming from a vertex $v_{i}$ for which $m_{i}+d_{i}>0$, and one right-handed Dehn twist about each boundary component coming from a vertex for which $m_{i}+d_{i}<0$. 
Theorem 1.3 Given any plumbing graph $G$, let $X(G)$ be the associated plumbed 4-manifold. Then $\partial X$ has an open book decomposition with page $\Sigma(G)$ and monodromy $h(G)$.

In the case where $m_{i}+d_{i}=0$ for all vertices, this is in fact giving us a fibration of $\partial X$ over $S^{1}$, or an "open book with empty binding". If we apply theorem 1.3 to a positive symplectic configuration graph, the reader may notice that the monodromy as described here is the inverse of the monodromy as described in theorem 1.1; this is because here we are describing $\partial X$ whereas in theorem 1.1 we are describing $-\partial X$.

The author would like to thank A. Stipsicz for suggesting the idea of trying to understand boundary behaviors for neighborhoods of symplectic configurations as a way to search for new symplectic surgeries, and would like to thank A. Stipsicz, G. Matic, M. Symington and R. Kirby for helpful discussions and for looking at drafts of this paper and suggesting improvements.

\section{Applications}

Before presenting the main proofs we investigate a few consequences of theorem 1.1 and point out some directions in which to look for further applications.

Given a compact surface $\Sigma$, we say that a mapping class $h \in \mathcal{M}(\Sigma)$ is positive if $h$ can be expressed as a product of right-handed Dehn twists. It is not hard to show, using compact Stein surfaces and Legendrian surgeries, that if $h \in \mathcal{M}(\Sigma)$ is positive then $\mathcal{B}(\Sigma, h)$ is strongly symplectically fillable (see [7], [1] and [3]).

Given a configuration graph $G$, let $Q(G)$ be the associated intersection form; i.e. $Q(G)=\left(q_{i j}\right)$, where $q_{i i}=m_{i}$ and $q_{i j}$ is the number of edges connecting $v_{i}$ to $v_{j}$. Let $b^{+}(G)$ denote the number of positive eigenvalues of $Q(G)$.

The following is a straightforward application of the adjunction inequality (see [9]):

Corollary 2.1 Let $G$ be a connected positive graph with $b^{+}(G)>1$ and with at least one vertex $v_{i}$ for which $m_{i}>2 g_{i}-2$. Then $\mathcal{B}(\Sigma(G), h(G))$ is not strongly symplectically fillable and therefore $h(G)$ is not positive in $\mathcal{M}(\Sigma(G))$.

Proof of corollary 2.1 The symplectic manifold $(N(G), \omega(G))$ constructed in theorem 1.1 is open; a function $F<f$ on $M(G)$ gives a compact version $\left(N_{F}(G), \omega(G)\right)$ where $N_{F}(G)=\phi\{(t, p) \mid F(p) \leq t<f(p)\} \cup C(G)$. Suppose 
that $\mathcal{B}(\Sigma(G), h(G))$ is strongly symplectically fillable. Then there exists a closed symplectic 4 -manifold $(X, \omega)$ containing $\left(N_{F}(G), \omega(G)\right)$ for some function $F<$ $f$ on $M(G)$ and containing the configuration $C(G)=\Sigma_{1} \cup \ldots \cup \Sigma_{n}$. The intersection form for $N_{F}(G)$ is $Q(G)$; since $b^{+}(G)>1$ we know that $b_{2}^{+}(X)>$ 1. Thus the adjunction inequality applies, which states that, for any closed surface $\Sigma \subset X$ (with $[\Sigma]$ not torsion in $H_{2}(X)$ if genus $\left.(\Sigma)=0\right),\left|c_{1}(\omega) \cdot \Sigma\right|+$ $\Sigma \cdot \Sigma \leq 2$ genus $(\Sigma)-2$. However, here we have an embedded surface $\Sigma_{i}$ for which $\Sigma_{i} \cdot \Sigma_{i}>2$ genus $\left(\Sigma_{i}\right)-2$, which is a contradiction.

Remark 2.2 For any surface $\Sigma$, let us call a relation in $\mathcal{M}(G)$ of the form $\delta=$ $w$ a boundary-interior relation if $\delta$ is a single right twist about each boundary component and $w$ is some word in interior right twists. We have the following trivial observation: For a given $G, h(G)$ is positive if and only if there exists a boundary-interior relation $\delta(G)=w$ in $\mathcal{M}(\Sigma(G))$ such that the word $w$ includes all the twists in $\sigma(G)$. (The order in which the twists of $\sigma(G)$ appear in $w$ does not matter.) Boundary-interior relations have a variety of uses, including giving constructions of topological Lefschetz pencils (see [3]).

Let $\Sigma_{g}^{n}$ denote a surface of genus $g$ with $n$ boundary components. Two boundary-interior relations are the "lantern relation" in $\mathcal{M}\left(\Sigma_{0}^{4}\right)$ and the "chain relation" in $\mathcal{M}\left(\Sigma_{g}^{2}\right)$ (see [14]). Figure 2 shows a set of example graphs $G_{1}, G_{2}$, $G_{3}, G_{4}$ on the left (here we have suppressed the areas and only given the pair $(g, m)$ at each vertex $)$, with the associated surfaces $\Sigma\left(G_{1}\right), \ldots, \Sigma\left(G_{4}\right)$ drawn on the right. The lantern relation shows that $h\left(G_{1}\right)$ is positive, while corollary 2.1 tells us that $h\left(G_{2}\right)$ is not positive. Thus there does not exist a lantern-type relation on $\Sigma_{0}^{5}=\Sigma\left(G_{2}\right)$. The chain relation shows that $h\left(G_{3}\right)$ is positive (where $G_{3}$ has $g+1$ edges between 2 vertices so that $\left.\Sigma\left(G_{3}\right)=\Sigma_{g}^{2}\right)$. The graph $G_{4}$ also has $g+1$ edges connecting two vertices, but now the self-intersections are arbitrary integers $a$ and $b$ such that $a+g+1>0$ and $b+g+1>0$. If $a$ and $b$ are positive and $a b>(g+1)^{2}$, then $b^{+}\left(G_{4}\right)=2$ and corollary 2.1 tells us that $h\left(G_{4}\right)$ is not positive. Thus, if $a b>(g+1)^{2}, A=a+g+1, B=b+g+1$ and $n=A+B$, then there cannot exist a boundary-interior relation $\delta=w$ in $\mathcal{M}\left(\Sigma_{g}^{n}\right)$ if $w$ contains twists along $g+1$ disjoint curves which, collectively, separate $\Sigma_{g}^{n}$ into two genus 0 pieces one containing $A$ of components of $\partial \Sigma_{g}^{n}$ and the other containing $B$ components.

The existence of an elliptic Lefschetz pencil on $\mathbb{C P}^{2}$ with 9 points in the base locus and 12 singular fibers means that there exists a boundary-interior relation $\delta=w$ in $\mathcal{M}\left(\Sigma_{1}^{9}\right)$ such that $w$ is the product of twists along 12 curves $C_{1}, \ldots, C_{12}$. This author is not aware that this relation has been written down and has been curious for a long time as to where these 12 curves are. We do 

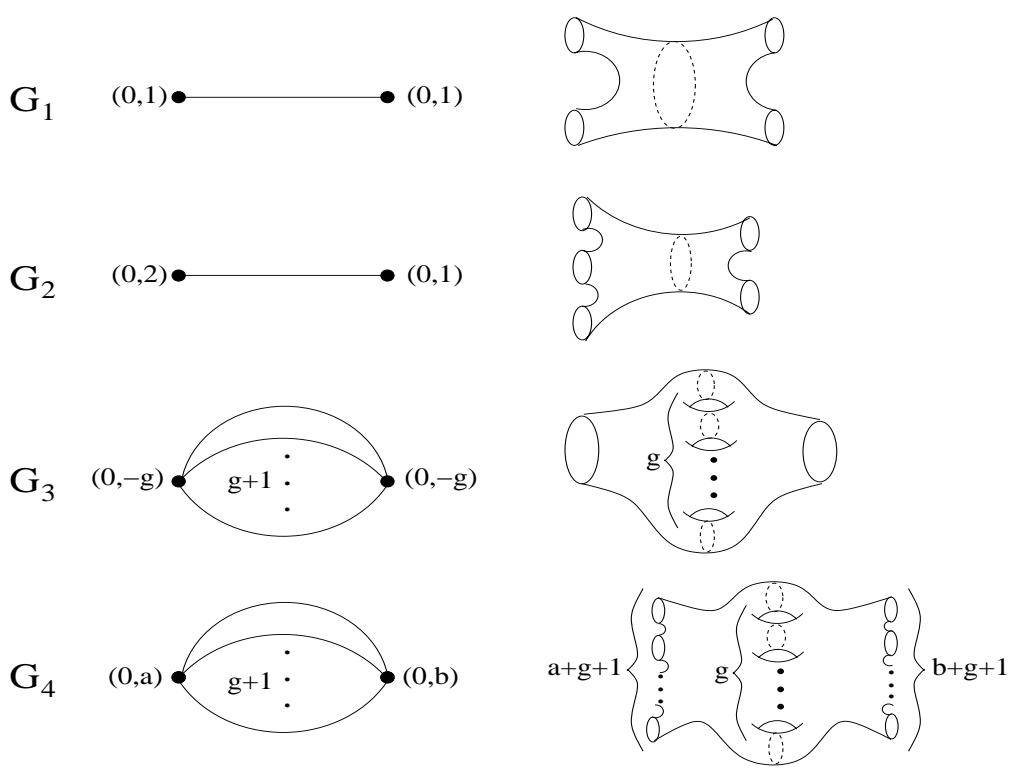

Figure 2: Four example graphs for remark 2.2

know that when we blow up the 9 points we get a Lefschetz fibration given by the relation $(a b)^{6}=1$ in $\mathcal{M}\left(\Sigma_{1}^{0}\right)$, where $a$ is a meridinal right twist and $b$ is a longitudinal right twist, so that, after embedding $\Sigma_{1}^{9}$ in $\Sigma_{1}^{0}$ the odd $C_{i}$ 's are isotopic to meridians and the even $C_{i}$ 's are isotopic to longitudes. Corollary 2.1 gives a little more information, ruling out certain possible arrangements of curves. For example, the observation from the previous paragraph about $G_{4}$ shows that no two of the curves may be disjoint and separate 4 boundary components from the other 5 . Other possibilities can be ruled out by considering various cycle graphs.

Along much the same lines, we have:

Corollary 2.3 Let $G_{1}$ and $G_{2}$ be positive, connected symplectic configuration graphs with $b^{+}\left(G_{1}\right)>0$ and $b^{+}\left(G_{2}\right)>0$, with at least one vertex $v_{i}$ in $G_{1}$ for which $m_{i}>2 g_{i}-2$. Then, although each $\mathcal{B}\left(\Sigma\left(G_{i}\right), h\left(G_{i}\right)\right)$ may be strongly symplectically fillable, there does not exist a connected symplectic 4-manifold with disconnected convex boundary $\mathcal{B}\left(\Sigma\left(G_{1}\right), h\left(G_{1}\right)\right) \amalg \mathcal{B}\left(\Sigma\left(G_{2}\right), h\left(G_{2}\right)\right)$.

Proof If such a symplectic 4-manifold existed then there would exist a closed, connected, symplectic 4-manifold $(X, \eta)$ containing

$$
(N, \omega)=\left(N_{F_{1}}\left(G_{1}\right), \omega\left(G_{1}\right)\right) \amalg\left(N_{F_{2}}\left(G_{2}\right), \omega\left(G_{2}\right)\right)
$$


for appropriate functions $F_{1}$ and $F_{2}$. The intersection form for $N$ is $Q\left(G_{1}\right) \oplus$ $Q\left(G_{2}\right)$, so that $b_{2}^{+}(N)>1$; the rest of the contradiction is identical to that in the preceding proof.

Remark 2.4 McDuff [8] has shown that symplectic 4-manifolds with disconnected convex boundary do exist. By a result of Gromov [6] (made explicit in [2] and [8]), it is not possible to have multiple convex $S^{3}$ boundary components, which is the case of this corollary when $\Sigma\left(G_{1}\right)$ and $\Sigma\left(G_{2}\right)$ are both disks. We hope that our result significantly enlarges the class of pairs of contact manifolds which cannot appear as disconnected convex boundaries, despite being individually strongly symplectically fillable. It is not hard to construct examples of graphs $G$ with $b^{+}(G)=1$, with one vertex for which $m_{i}>2 g_{i}-2$ and such that $M(G)$ is not $S^{3}$, but it is then not necessarily clear how to show that $(M(G), \xi(G))$ is in fact strongly symplectically fillable.

It would be interesting to construct arguments in the opposite direction:

Question 2.5 Are there any positive configuration graphs $G$ for which we can show directly that $\mathcal{B}(\Sigma(G), h(G))$ is overtwisted and hence conclude, without appealing to the adjunction inequality, that a symplectic configuration with configuration graph $G$ cannot embed in a closed symplectic 4-manifold?

Showing that $\mathcal{B}(\Sigma, h)$ is overtwisted for a given surface $\Sigma$ and mapping class $h \in$ $\mathcal{M}(\Sigma)$ is, in principle, purely a mapping class group problem, as follows: Let $\Sigma$ and $\Sigma^{\prime}$ be compact surfaces with boundary and $h \in \mathcal{M}(\Sigma)$ and $h^{\prime} \in \mathcal{M}\left(\Sigma^{\prime}\right)$ be mapping classes. We say that $\left(\Sigma^{\prime}, h^{\prime}\right)$ is a positive (resp. negative) stabilization of $(\Sigma, h)$ if $\Sigma^{\prime}$ is the result of attaching a 1 -handle to $\Sigma$ and $h^{\prime}=h \circ \tau$, where $\tau$ is a right-handed (resp. left-handed) Dehn twist along a curve dual to the co-core of the 1-handle. It can be shown, using results of Giroux [5] and Torisu [13], that $\mathcal{B}(\Sigma, h)$ is overtwisted if and only if there exist pairs $\left(\Sigma^{\prime}, h^{\prime}\right)$ and $\left(\Sigma^{\prime \prime}, h^{\prime \prime}\right)$ such that $\left(\Sigma^{\prime}, h^{\prime}\right)$ is related to $(\Sigma, h)$ by a sequence of positive stabilizations and destabilizations and $\left(\Sigma^{\prime}, h^{\prime}\right)$ is a negative stabilization of $\left(\Sigma^{\prime \prime}, h^{\prime \prime}\right)$

Lastly, we point out that theorem 1.1 could be used to produce new symplectic surgeries. If, for a given symplectic configuration graph $G$, we can find some other interesting symplectic 4 -manifold $(Y, \eta)$ with concave boundary contactomorphic to $(-M(G), \xi(G))$, then we may surger out a neighborhood of a configuration $C$ for which $G(C)=G$ and replace it with $(Y, \eta)$. (Symington $[10,11]$ has investigated configurations which have neighborhoods with convex boundaries, and has used this to produce useful symplectic surgeries; this paper is partly inspired by that work.) 
Question 2.6 Given $G$, is there any canonical way to produce such a $(Y, \eta)$ with significantly different topology from $(N(G), \omega(G))$ ? Especially interesting would be examples where $Y$ is a rational homology ball.

\section{The main proof}

Proof of theorem 1.1 Our proof is a three step construction. In "Step 1" we construct a symplectic $4-$ manifold $(X, \omega)$ with a symplectic dilation (Liouville vector field) $V^{+}$defined on all of $X$ and pointing out along $\partial X . X$ will contain a configuration $Z$ of properly embedded symplectic surfaces with boundary, which will become the configuration $C(G)$ once we cap off the surfaces with 2-handles. In "Step 2", we arrange that the induced positive contact form on $\partial X$ has a particularly nice form and then we produce a symplectic contraction $V^{-}$defined on $X \backslash Z$, pointing out along $\partial X \backslash \partial Z$. In "Step 3" we cap off the surfaces in $Z$ by attaching appropriately framed 4-dimensional symplectic 2handles along $\partial Z$; these handles have the effect of turning the convex boundary into a concave boundary. The symplectic contraction $V^{-}$from Step 2 extends across the 2-handles; flow along $-V^{-}$gives the symplectomorphism $\phi$ (after attaching an open collar to the boundary).

Step 1 For lack of a better term, the objects we construct in this step will be called "Step 1 objects". A Step 1 object is a 6 -tuple $\left(X, \omega, Z, V^{+}, f, p\right)$ where:

- $(X, \omega)$ is a compact symplectic 4-manifold with boundary.

- $Z=F_{1} \cup \ldots \cup F_{n}$ is a configuration of symplectic surfaces with boundary, each properly embedded in $X$, with $\omega$-orthogonal intersections.

- $f$ is a proper Morse function on $X$ which restricts to each $F_{i}$ as a proper Morse function, with only critical points of index 0 and 1 , all of which lie in $Z$.

- $V^{+}$is a symplectic dilation on $(X, \omega)$, tangent to $Z$ and gradient-like for $f$, inducing a positive contact structure $\xi^{+}=\operatorname{ker}\left(\left.\imath_{\left(V^{+}\right)} \omega\right|_{\partial X}\right)$ on $\partial X$.

- $p: \partial X \backslash \partial Z \rightarrow S^{1}$ is a fibration such that $(\partial Z, p)$ is an open book on $\partial X$.

- $\xi^{+}$is supported by $(\partial Z, p)$.

Each component $K$ of $\partial F_{i} \subset \partial Z$ has two different natural framings, the framing coming from the page of the open book, which is a Seifert surface for $\partial Z$, and the framing coming from a Seifert surface for $\partial F_{i}$. Denote the former 
framing $\operatorname{pf}(K)$ (for "page framing") and denote the latter framing $\operatorname{cf}(K)$ (for "component framing").

Our goal is to produce a Step 1 object $\left(X, \omega, Z, V^{+}, f, p\right)$ related to the given graph $G$ as follows:

- $Z=F_{1} \cup \ldots \cup F_{n}$, where each surface $F_{i}$ corresponds to a vertex $v_{i}$ in $G$ and the intersections correspond to the edges in $G$.

- $\operatorname{genus}\left(F_{i}\right)=g_{i}$

- $\partial F_{i}$ has $m_{i}+d_{i}$ components.

- For each $F_{i}$, there is one component $K$ of $\partial F_{i}$ for which $\operatorname{pf}(K)=\operatorname{cf}(K)-$ $d_{i}$, and for all other components the two framings are equal.

Topologically $X$ is built from 0 -handles and 1 -handles, with one 0 -handle for each vertex and each edge in $G$, and with a 1 -handle connecting an edge 0 -handle to a vertex 0 -handle if that edge is incident with that vertex.

We begin with two basic Step 1 objects $A=\left(X_{A}, \omega_{A}, Z_{A}, V_{A}^{+}, f_{A}, p_{A}\right)$ and $B=\left(X_{B}, \omega_{B}, Z_{B}, V_{B}^{+}, f_{B}, p_{B}\right)$ defined as follows (here we use polar coordinates $\left(r_{1}, \theta_{1}, r_{2}, \theta_{2}\right)$ on $\left.\mathbb{R}^{4}\right)$ :

- $X_{A}=X_{B}=B^{4}=\left\{r_{1}^{2}+r_{2}^{2} \leq 1\right\} \subset \mathbb{R}^{4}$.

- $\omega_{A}=\omega_{B}=r_{1} d r_{1} d \theta_{1}+r_{2} d r_{2} d \theta_{2}$.

- $Z_{A}=\left\{r_{2}=0\right\}$ and $Z_{B}=\left\{r_{2}=0\right\} \cup\left\{r_{1}=0\right\}$.

- $V_{A}^{+}=V_{B}^{+}=\frac{1}{2}\left(r_{1} \partial_{r_{1}}+r_{2} \partial_{r_{2}}\right)$.

- $f_{A}=f_{B}=r_{1}^{2}+r_{2}^{2}$.

- $p_{A}=\theta_{2}$ while $p_{B}=\theta_{1}+\theta_{2}$.

Thus $\left(\partial Z_{A}, p_{A}\right)$ is the standard open book on $S^{3}$ with page equal to a disk and binding the unknot, and $\left(\partial Z_{B}, p_{B}\right)$ is the open book on $S^{3}$ with page equal to an annulus (a left-twisted Hopf band), monodromy equal to a single right twist about the core circle of the annulus, and the Hopf link with positive linking number as the binding. Note that, for the single component of $\partial Z_{A}$, we have $\mathrm{pf}=\mathrm{cf}$, whereas for each of the two components of $\partial Z_{B}$, we have $\mathrm{pf}=\mathrm{cf}-1$.

We think of these two objects as 4-dimensional symplectic 0 -handles, in the sense of Weinstein [15]. We also have Weinstein's 4-dimensional symplectic 1 -handle, which is constructed as a neighborhood of the origin in $\mathbb{R}^{4}$ with the standard symplectic form $\omega=d x_{1} d y_{1}+d x_{2} d y_{2}$, the Morse function $f=-x_{1}^{2}+$ $y_{1}^{2}+x_{2}^{2}+y_{2}^{2}$ and the symplectic dilation $V^{+}=-x_{1} \partial_{x_{1}}+2 y_{1} \partial_{y_{1}}+\frac{1}{2}\left(x_{2} \partial_{x_{2}}+y_{2} \partial_{y_{2}}\right)$. Weinstein shows that we can always attach such a 1 -handle at any two points on 


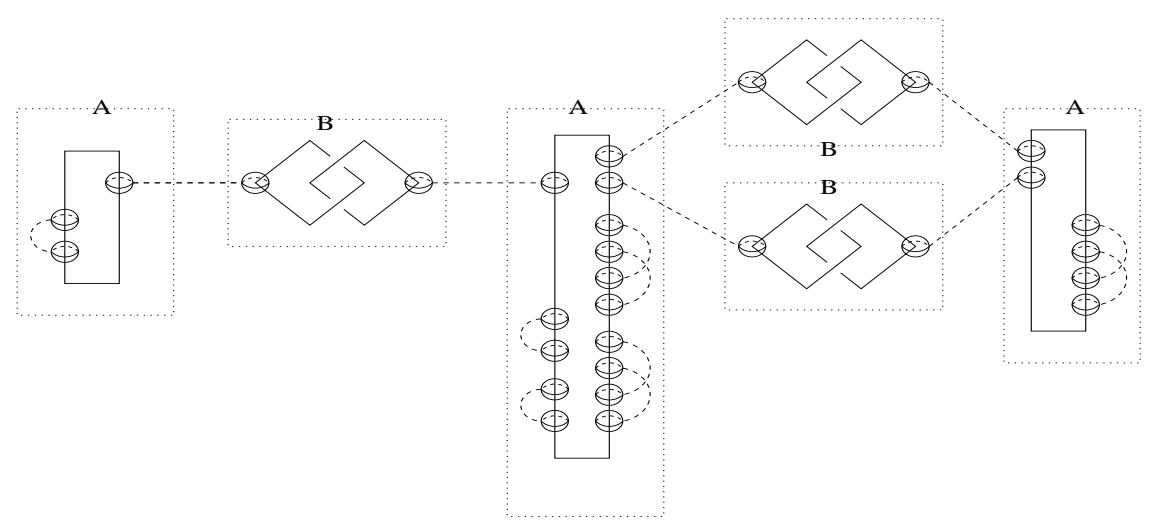

Figure 3: The Step 1 object corresponding to the graph $G$ in figure 1

a convex boundary of a symplectic 4-manifold, such that the symplectic forms and symplectic dilations match up along the glueing. In [3] we have shown that, if in addition the contact structure on the boundary is supported by an open book and the attaching 3 -balls of the 1 -handle are pierced by the binding (as the $z$-axis pierces the unit ball in $\mathbb{R}^{3}$ ), then the handle can be constructed in such a way that the new contact structure produced by the associated contact surgery is also supported by an open book. The new page is produced from the old page by attaching a 2-dimensional 1 -handle at the corresponding intervals along the binding and the new monodromy is equal to the old monodromy extended by the identity on the 1 -handle. (Note that the 2-dimensional 1handle is explicitly the set $\left\{x_{2}=y_{2}=0\right\}$ inside the 4-dimensional 1-handle, and that this a symplectic surface.)

Now suppose that we are attaching such a 1 -handle to a Step 1 object $\left(X, \omega, Z, V^{+}, f, p\right)$ at two points along the binding $\partial Z$ (not connecting two surfaces in $Z$ that already intersect). Then we produce a new Step 1 object $\left(X_{1}, \omega_{1}, Z_{1}, V_{1}^{+}, f_{1}, p_{1}\right)$, where $Z_{1}$ is the result of attaching a 1 -handle to $Z$, $\left(\partial Z_{1}, p_{1}\right)$ is the open book described in the preceding paragraph, and $f_{1}$ has a single new index 1 critical point.

We now describe how to build our desired Step 1 object corresponding to the given graph $G$. Figure 3 illustrates this construction applied to the graph $G$ of figure 1. Start with a Step 1 object which is a disjoint union of many copies of the 0 -handle objects $A$ and $B$, with one $A$ for each vertex and one $B$ for each edge in $G$. These are indicated in figure 3 by dotted rectangles, with the bindings of the open books indicated as solid links (unknots or Hopf links). 


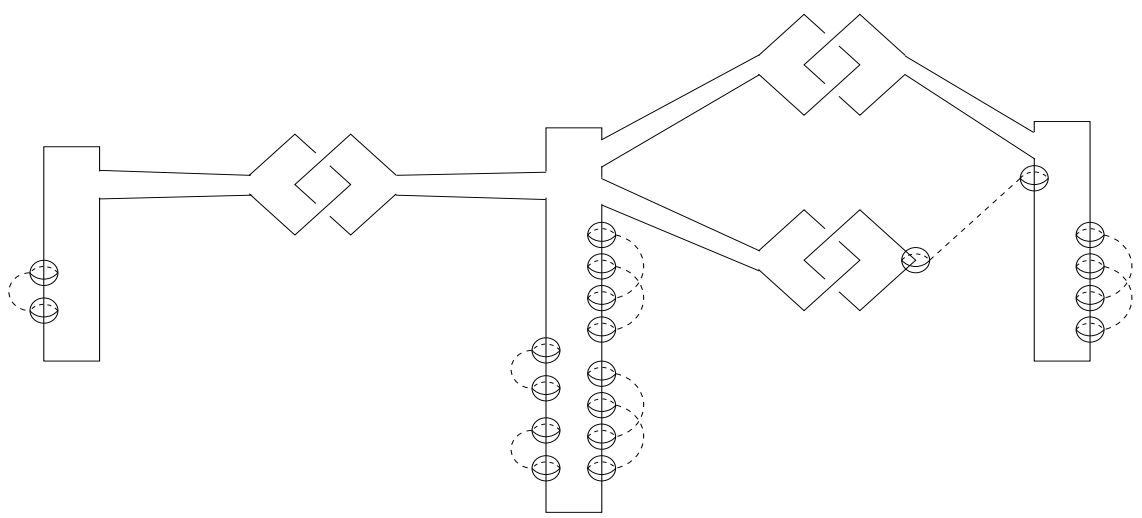

Figure 4: The same Step 1 object, drawn as a Kirby calculus diagram in a single $S^{3}$

For an edge $e_{k}$ connecting two vertices $v_{i}$ and $v_{j}$, let $B_{k}$ be the corresponding copy of $B$ and $A_{i}$ and $A_{j}$ be the corresponding copies of $A$. Connect $B_{k}$ to $A_{i}$ by a symplectic 1 -handle as described above, with the 1 -handle connecting one component of the binding in $B_{k}$ to the binding in $A_{i}$, and connect $B_{k}$ to $A_{j}$ by a 1 -handle connecting the other component of the binding in $B_{k}$ to the binding in $A_{j}$. In figure 3 we have drawn the attaching 3 -balls of these 1-handles with dashed lines connecting them. After doing this for all the edges, we have a Step 1 object where the configuration $Z$ is a configuration of disks, one disk for each vertex, with intersections given by $G$. Furthermore, for each component $K_{i}$ of $\partial Z$ corresponding to a vertex $v_{i}$, we have $\operatorname{pf}\left(K_{i}\right)=$ $\operatorname{cf}\left(K_{i}\right)-d_{i}$. Now attach $2 g_{i} 1$-handles in pairs along each component $K_{i}$ of $\partial Z$ to get a Step 1 object for which the configuration is now a configuration of surfaces $Z=F_{1} \cup \ldots \cup F_{n}$ where each $F_{i}$ corresponds to a vertex $v_{i}$, each $F_{i}$ has a single boundary component, and $\operatorname{genus}\left(F_{i}\right)=g_{i}$, with the intersections given by $G$. These pairs of 1 -handles are drawn on the lower right of each $A$ binding. We still have $\operatorname{pf}\left(\partial F_{i}\right)=\operatorname{cf}\left(\partial F_{i}\right)-d_{i}$. Finally, attach $\left(m_{i}+d_{i}-1\right)$ 1 -handles along an isolated stretch of $\partial F_{i}$ to get $m_{i}+d_{i}$ binding components for each $F_{i}$; for the "new" binding components we will have $\mathrm{pf}=\mathrm{cf}$, while one binding component still has $\mathrm{pf}=\mathrm{cf}-d_{i}$. These 1-handles are drawn on the lower left of each $A$ binding. Figure 4 shows a standard Kirby calculus diagram for the same construction, drawn in a single copy of $S^{3}$; the link drawn is the binding of an open book, not a surgery link (yet).

Note that the page of the resulting open book $(\partial C, p)$ in the final Step 1 object $\left(X, \omega, Z, V^{+}, f, p\right)$ is exactly the surface $\Sigma(G)$ associated to the graph $G$ and that the monodromy is exactly the mapping class $\sigma(G)$. Thus $\left(\partial X, \xi^{+}\right)=$ 
$\mathcal{B}(\Sigma(G), \sigma(G))$.

Step 2 For each $F_{i}$ in $Z=F_{1} \cup \ldots \cup F_{n}$, choose a positive constant $b_{i}<$ $a_{i} /\left(2 \pi\left(m_{i}+d_{i}\right)\right)$. By an explicit construction (similar to that in section 4 of [3]) one can show that there exists a positive contact form $\alpha^{+}$supported by $(\partial Z, p)$ with the following behavior near each component $K$ of each $F_{i}$ : In a neighborhood of $K$ there should exist solid torus coordinates $(r, \mu, \lambda$ ) (radial, meridinal and longitudinal coordinates, with $K=\{r=0\}$ ) with respect to which $\alpha^{+}=\frac{1}{2} r^{2}(d \mu-d \lambda)+b_{i} d \lambda$ and $p=\mu+\lambda$. After an isotopy fixing $\partial Z$, by theorem 1.2, we can assume that $\operatorname{ker} \alpha^{+}=\xi^{+}=\operatorname{ker}\left(\left.\imath_{\left(V^{+}\right)} \omega\right|_{\partial X}\right)$. Because $V^{+}$is defined on all of $X$ and is gradient-like for $f$, we can use the canonical symplectification of $\xi^{+}$and enlarge and/or trim $X$ so that in fact $\alpha^{+}=\left.\imath_{\left(V^{+}\right)} \omega\right|_{\partial X}$.

At this point note that the area of each $F_{i}$ is $\int_{F_{i}} \omega=\int_{\partial F_{i}} \alpha^{+}=2 \pi\left(m_{i}+d_{i}\right) b_{i}<$ $a_{i}$.

Now we recall some definitions from [4]. A contact pair on a 3-manifold $M$ is a pair $\left(\alpha^{+}, \alpha^{-}\right)$of 1 -forms defined, respectively, on open subsets $M^{ \pm}$with $M=M^{+} \cup M^{-}$, such that $\pm \alpha^{ \pm} \wedge d \alpha^{ \pm}>0$ on $M^{ \pm}$and such that $d \alpha^{+}=-d \alpha^{-}$ on $M^{0}=M^{+} \cap M^{-}$. In particular $\alpha^{+}$(resp. $\alpha^{-}$) is a positive (resp. negative) contact form and $\alpha^{0}=\alpha^{+}+\alpha^{-}$is a closed, nowhere zero 1 -form on $M^{0}=$ $M^{+} \cap M^{-}$. A dilation-contraction pair on a symplectic 4 -manifold $(X, \omega)$ is a pair $\left(V^{+}, V^{-}\right)$defined, respectively, on open subsets $X^{ \pm} \subset X$, such that $\mathcal{L}_{\left(V^{ \pm}\right)} \omega= \pm \omega$ and $\omega\left(V^{+}, V^{-}\right)=0$. We say that $\left(V^{+}, V^{-}\right)$transversely covers a 3-dimensional submanifold $M$ if $M \subset X^{+} \cup X^{-}$and both $V^{+}$and $V^{-}$are positively transverse to $M$. This gives us an induced contact pair $\left(\alpha^{+}, \alpha^{-}\right)$on $M$ defined by $\alpha^{ \pm}=\left.\imath_{\left(V^{ \pm}\right)} \omega\right|_{M}$, with domains $M^{ \pm}=M \cap X^{ \pm}$.

In our situation we have the positive contact form $\alpha^{+}$defined on $(\partial X)^{+}=\partial X$, supported by $(\partial Z, p)$; for a large enough constant $k>0,\left(\alpha^{+}, \alpha^{-}=k d p-\alpha^{+}\right)$ will be a contact pair (with $(\partial X)^{-}=\partial X \backslash \partial Z$ ). Fix such a $k$ and henceforth let $\alpha^{-}=k d p-\alpha^{+}$.

Backward flow along the symplectic dilation $V^{+}$starting on $\partial X$ gives us an embedding $\phi^{+}:(-\infty, 0] \times \partial X \hookrightarrow X$ such that $\phi^{+}(0, p)=p, D \phi^{+}\left(\partial_{t}\right)=V^{+}$ and $\left(\phi^{+}\right)^{*} \omega=d\left(e^{t} \alpha^{+}\right)$, where $t$ is the coordinate on $(-\infty, 0]$. Since $V^{+}$is tangent to $Z$ and gradient-like for $f$, we know that $\phi^{+}((-\infty, 0] \times(\partial X \backslash \partial Z))=$ $X \backslash Z$. Lemma 4.1 in [4] then tells us that there exists a unique symplectic contraction $V^{-}$defined on $X \backslash Z$ such that $\left(V^{+}, V^{-}\right)$is a dilation-contraction pair transversely covering $\partial X$ inducing the contact pair $\left(\alpha^{+}, \alpha^{-}\right)$. Forward flow along $-V^{-}$starting on $\partial X \backslash \partial Z$ then gives an embedding $\phi^{-}$from $\{(t, p) \mid 0 \leq$ $t<F(p)\} \subset \mathbb{R} \times(-(\partial X \backslash \partial Z))$ into $X$ for some function $F: \partial X \backslash \partial Z \rightarrow(0, \infty]$, 
such that $\phi^{-}(0, p)=p, D \phi^{-}\left(\partial_{t}\right)=-V^{-}$and $\left(\phi^{-}\right)^{*} \omega=d\left(e^{t} \alpha^{-}\right)$. The proof of lemma 4.1 in [4] shows us how to explicitly calculate $V^{-}$given $\left(\alpha^{+}, \alpha^{-}\right)$, from which we can see that, in our case, $F<\infty$ and the image of $\phi^{-}$is all of $X \backslash Z$, with $Z \backslash \partial Z=\left\{\lim _{t \rightarrow F(p)} \phi^{-}(t, p) \mid p \in \partial X \backslash \partial Z\right\}$.

Step 3 Our final symplectic 4 -manifold $(N(G), \omega(G))$ will be constructed by attaching a symplectic 2 -handle as described in [4] along each component $K$ of the binding $\partial Z \subset \partial X$ with framing pf $(K)+1$, and then attaching an open symplectic collar on the boundary. In [4] it is shown that one can attach handles in this way to produce a concave boundary, and in [3], it is shown that the contact manifold on the boundary is indeed $\mathcal{B}\left(\Sigma(G), \sigma(G)^{-1} \circ \delta(G)\right)$. It is not hard to see that the 4-manifold produced in this way is a neighborhood of a configuration of closed surfaces with the correct genera and intersecting according to the graph $G$; the surfaces are simply the surfaces $F_{i}$ in $Z$ capped off with the descending disks of the 4-dimensional 2-handles.

To see that the self-intersections are correct, note that the self-intersection of a surface $\Sigma_{i}$ built by attaching 2 -handles, framed as above, along $\partial F_{i}$ for one of the surfaces $F_{i} \subset Z$ is equal to the sum over all components $K$ of $\partial F_{i}$ of $(\operatorname{pf}(K)+1)-\operatorname{cf}(K)$, which is exactly $m_{i}$.

To see that the remaining claims of the theorem hold, we need to look more closely at the structure of the 2-handles. Proposition 4.6 in [4] shows us how to construct our 2-handles; here we give the construction tailored to the special case at hand. For each $F_{i} \subset Z$, let $c_{i}=a_{i} /\left(2 \pi k\left(m_{i}+d_{i}\right)\right.$ ) (with $k$ as fixed in Step 2). The handle $H$ that will attach to each component $K$ of $\partial F_{i}$ is a subset of $\mathbb{R}^{4}$ with the symplectic form $\omega_{H}=c_{i}\left(r_{1} d r_{1} d \theta_{1}+r_{2} d r_{2} d \theta_{2}\right)$ with the Morse function $f_{H}=-r_{1}^{2}+r_{2}^{2}$. The following is a dilation-contraction pair on $\left(\mathbb{R}^{4}, \omega_{H}\right)$ :

$$
\begin{gathered}
V_{H}^{+}=\left(\frac{1}{2} r_{1}-\frac{k}{r_{1}}\right) \partial_{r_{1}}+\frac{1}{2} r_{2} \partial_{r_{2}} \\
V_{H}^{-}=-\frac{1}{2} r_{1} \partial_{r_{1}}-\left(\frac{1}{2} r_{2}-\frac{k}{r_{2}}\right) \partial_{r_{2}}
\end{gathered}
$$

$\left(V_{H}^{+}, V_{H}^{-}\right)$transversely covers the regular level sets of $f$ as long as $-2 k<$ $f<2 k$. Let $\epsilon_{1}=2\left(b_{i} / c_{i}-k\right)$; note that $-2 k<\epsilon_{1}<0$. Choose some $\epsilon_{2}$ with $0<\epsilon_{2}<2 k$. Construct $H$ so that the attaching boundary of $H$ (which we call $\left.\partial_{1} H\right)$ is a neighborhood of $\left\{r_{2}=0\right\}$ in $f^{-1}\left(\epsilon_{1}\right)$ and so that the free boundary $\partial_{2} H$ interpolates from a neighborhood of $\left\{r_{1}=0\right\}$ in $f^{-1}\left(\epsilon_{2}\right)$ down to $f^{-1}\left(\epsilon_{1}\right)$, so that both boundaries are transverse to both $V_{H}^{+}$and $V_{H}^{-}$(where defined). (See section 2 of [4] for a more detailed discussion of this type of 
handle construction and notation.) On $\partial_{1} H$, we use solid torus coordinates $\left(r=\sqrt{c_{i}} r_{2}, \mu=\theta_{2}, \lambda=-\theta_{1}\right)$; the contact pair induced by $\left(V_{H}^{+}, V_{H}^{-}\right)$on $\partial_{1} H$ then becomes:

$$
\left(\alpha_{H}^{+}=\frac{1}{2} r^{2}(d \mu-d \lambda)+b_{i} d \lambda, \alpha_{H}^{-}=k(d \mu+d \lambda)-\alpha_{H}^{+}\right)
$$

This is exactly the contact pair we have on a neighborhood of $K \subset \partial X$, so that lemma 4.1 in [4] tells us we can attach $H$ to $X$ by identifying the $(r, \mu, \lambda)$ coordinates on $\partial_{1} H$ with the $(r, \mu, \lambda)$ coordinates in a neighborhood of $K$, in such a way that the symplectic forms and the dilation-contraction pairs fit together smoothly. Note that since $p=\mu+\lambda$ in a neighborhood of $K$, we are attaching $H$ with framing $\mathrm{pf}(K)+1$.

After attaching these handles to each component of $\partial F_{i}$, we get a closed surface $\Sigma_{i}$ which is the union of $F_{i}$ and the disks $D=\left\{r_{2}=0\right\} \cap H=\left\{r_{2}=0, r_{1}^{2} \leq\right.$ $\left.2\left(k-b_{i} / c_{i}\right)\right\}$ in each handle $H . \Sigma_{i}$ is smooth because $V^{+}$is tangent to $F_{i}$ and $V_{H}^{+}$is tangent to $D . \Sigma_{i}$ is symplectic because $F_{i}$ and $D$ are both symplectic. We have already arranged that the area of $F_{i}$ is $2 \pi\left(m_{i}+d_{i}\right) b_{i}$. The area of each disk $D$ is $2 \pi c_{i}\left(k-b_{i} / c_{i}\right)$. Thus the area of $\Sigma_{i}$ is exactly $a_{i}$. Let $C(G)=\Sigma_{1} \cup \ldots \cup \Sigma_{n}$.

Note that, in the handle $H$, the symplectic contraction $V_{H}^{-}$is defined across all of the free boundary $\partial_{2} H$ whereas the dilation $V_{H}^{+}$does not extend across the ascending circle $\left\{r_{1}=0\right\} \cap \partial_{2} H$. Thus after attaching all the handles we get a symplectic $4-$ manifold $\left(X_{1}, \omega_{1}\right)$ with a dilation-contraction pair $\left(V_{1}^{+}, V_{1}^{-}\right)$ which transversely covers $\partial X_{1}$ inducing a contact pair $\left(\alpha_{1}^{+}, \alpha_{1}^{-}\right)$with domains $\left(\partial X_{1}\right)^{+}=\partial X_{1} \backslash L_{1}$ (where $L_{1}$ is the union of the ascending circles) and $\left(\partial X_{1}\right)^{-}=\partial X_{1}$. The closed 1 -form $\alpha_{1}^{0}=\alpha_{1}^{+}+\alpha_{1}^{-}$is $k d p_{1}$ for the natural open book $\left(L_{1}, p_{1}\right)$ on $\partial X_{1}$ that results from pf +1 surgeries on the binding of the open book $(\partial Z, p)$ on $\partial X$, the pages of which are still diffeomorphic to $\Sigma(G)$ and the monodromy of which is now $\sigma(G) \circ \delta(G)^{-1}$ (see [3]). The fact that $\left(\alpha_{1}^{+}, \alpha_{1}^{-}\right)$is a contact pair implies that $\alpha_{1}^{0} \wedge \alpha_{1}^{-}>0$, which in turn implies that $\alpha_{1}^{-}$, as a positive contact form on $-\partial X_{1}$, is supported by the open book $\left(L_{1},-p_{1}\right)$, which has page $\Sigma(G)$ and monodromy $\sigma(G)^{-1} \circ \delta(G)$. Thus we let $M(G)=-\partial X_{1}$ and $\xi(G)=\operatorname{ker} \alpha^{-}$. Also let $\alpha=\alpha^{-}$.

From the explicit form for $V_{H}^{-}$and the observations made in Step 2 about flow along $-V^{-}$, we see that flow along $-V_{1}^{-}$starting on $\partial X_{1}$ gives a diffeomorphism $\phi_{1}^{-}$from $\{(t, p) \mid 0 \leq t<f(p)\} \subset \mathbb{R} \times M(G)$ to $X_{1} \backslash C(G)$, for some function $f: M(G) \rightarrow(0, \infty)$, such that $\phi^{-}(0, p)=p, D \phi^{-}\left(\partial_{t}\right)=-V^{-}$, $\left(\phi^{-}\right)^{*} \omega_{1}=d\left(e^{t} \alpha\right)$ and $C(G)=\left\{\lim _{t \rightarrow f(p)} \phi^{-}(t, p) \mid p \in M(G)\right\}$.

Finally let $(N(G), \omega(G))=\left(X_{1}, \omega_{1}\right) \cup_{\phi^{-}}\left((-\infty, 0] \times M(G), d\left(e^{t} \alpha\right)\right)$; the embedding $\phi$ is simply $\phi^{-}$extended by the identity on $(-\infty, 0] \times M(G)$. 


\section{The nonpositive case}

If our initial graph $G$ is not positive, we can simply add extra vertices labelled $(0,0,1)$ to produce a graph $G^{\prime} \supset G$ which is positive. This corresponds to plumbing on some extra spheres of square 0 . We can now carry out the construction above applied to $G^{\prime}$, but stop short of attaching the 2-handles required to close off these extra spheres. This will give a model neighborhood $(N(G), \omega(G))$ of a configuration $C(G)$ with $G=G(C(G))$, but now the boundary will not be concave. Instead the boundary will be "partially convex and partially concave" in the following sense: $(N(G), \omega(G))$ will carry a dilation-contraction pair transversely convering $\partial N(G)$ inducing a contact pair $\left(\alpha^{+}, \alpha^{-}\right)$, but neither $\alpha^{+}$nor $\alpha^{-}$will be defined on all of $\partial N(G)$. Nevertheless, the pair $\left(\alpha^{+}, \alpha^{-}\right)$will determine the germ of $\omega(G)$ along $\partial N(G)$ and the dilation-contraction pair determines something like a canonical symplectification of the contact pair, so that we have good control on the symplectic topology of $N(G) \backslash C(G)$. Furthermore, $\left(\alpha^{+}, \alpha^{-}\right)$will be supported by a signed open book $\left(L^{+}, L^{-}, p\right)$ on $M=\partial N(G)$, by which we mean the following: $\left(L=L^{+} \amalg L^{-}, p\right)$ is an open book, $\alpha^{ \pm}$is defined on $M^{ \pm}=M \backslash L^{\mp}$, and $\alpha^{ \pm}$is supported, as a positive contact form on $\pm M^{ \pm}$, by $\left(L^{ \pm}, \pm p\right)$. The link $L^{-}$will be the union of the ascending circles for the 2 -handles that we did attach, while $L^{+}$will be the binding components from the Step 1 object to which we did not attach 2handles. Smaller or larger neighborhoods of $C(G)$ will have boundaries which are still transversely covered by the dilation-contraction pair and hence carry related contact pairs, all supported by the same signed open book. This line of reasoning will be investigated more thoroughly in a future paper.

\section{$5 \quad$ Boundaries of arbitrary plumbings}

Proof of theorem 1.3 If we strip the symplectic topology out from the proof of theorem 1.1, Step 2 is irrelevant. In Step 1, relabel $B$ as $B_{+}$and introduce a negative version of $B$ which we call $B_{-} ; B_{+}$(resp. $\left.B_{-}\right)$is a neighborhood of a positive (resp. negative) intersection of two disks, with a left-twisted (resp. right-twisted) Hopf band as the open book on the boundary, with monodromy equal to a single right (resp. left) Dehn twist along the core of the band. On $\partial B_{+}$we have $\mathrm{pf}=\mathrm{cf}-1$ and on $\partial B_{-}$we have $\mathrm{pf}=\mathrm{cf}+1$. Thus if we mimic 
the construction in theorem 1.1 but use copies of $B_{+}$for + edges and copies of $B_{-}$for - edges, we can produce a (non-symplectic) Step 1 object $(X, Z, f, p)$ where:

- $X$ is a compact $4-$ manifold with boundary which is a neighborhood of $Z$.

- $Z=F_{1} \cup \ldots \cup F_{n} \subset X$ is a configuration of properly embedded surfaces with boundary, corresponding to the vertices of $G$, with the appropriate genera and self-intersections and intersecting transversely according to the edges of $G$.

- $\partial F_{i}$ has $\left|m_{i}+d_{i}\right|$ components, unless $m_{i}+d_{i}=0$, in which case $\partial F_{i}$ has one component. (Recall that now $d_{i}$ is the signed count of edges connecting to $v_{i}$.)

- $p: \partial X \backslash \partial Z \rightarrow S^{1}$ is a fibration making $(\partial Z, p)$ an open book on $\partial X$.

- For each $F_{i}$, there is one component $K$ of $\partial F_{i}$ for which $\operatorname{pf}(K)=\operatorname{cf}(K)-$ $d_{i}$ and for all other components the two framings are equal.

When we get to Step 3, since we are no longer requiring that our handles be symplectic, we can attach 2 -handles along binding components with any framings we choose. Framing pf -1 produces a new open book with the same page and introduces a right-handed boundary Dehn twist into the monodromy; framing pf +1 also produces a new open book with the same page and introduces a left-handed boundary Dehn twist. Framing pf produces a new open book with the page alterred by capping off the corresponding boundary component, decreasing the number of binding components by 1 . For vertices $v_{i}$ with $m_{i}+d_{i}<0$, use (pf -1 )-framed binding handles, for vertices with $m_{i}+d_{i}=0$, use pf-framed binding handles and for vertices with $m_{i}+d_{i}>0$, use (pf +1$)$ framed binding handles. Because of these choices of framings we then calculate that for each $\Sigma_{i}, \Sigma_{i} \cdot \Sigma_{i}=m_{i}$.

\section{References}

[1] Selman Akbulut, Burak Ozbagci, On the topology of compact Stein surfaces, Int. Math. Res. Not. (2002) 769-782

[2] Yakov Eliashberg, Filling by holomorphic discs and its applications, from: "Geometry of low-dimensional manifolds, 2 (Durham, 1989)", Cambridge Univ. Press, Cambridge (1990) 45-67

[3] David T Gay, Explicit concave fillings of contact three-manifolds, Math. Proc. Cambridge Philos. Soc. 133 (2002) 431-441 
[4] David T Gay, Symplectic 2-handles and transverse links, Trans. Amer. Math. Soc. 354 (2002) 1027-1047 (electronic)

[5] Emmanuel Giroux, in preparation

[6] M Gromov, Pseudoholomorphic curves in symplectic manifolds, Invent. Math. 82 (1985) 307-347

[7] Andrea Loi, Riccardo Piergallini, Compact Stein surfaces with boundary as branched covers of $B^{4}$, Invent. Math. 143 (2001) 325-348

[8] Dusa McDuff, Symplectic manifolds with contact type boundaries, Invent. Math. 103 (1991) 651-671

[9] Dusa McDuff, Dietmar Salamon, Introduction to symplectic topology, second edition, Oxford University Press, Oxford (1998)

[10] Margaret Symington, Symplectic rational blowdowns, J. Differential Geom. 50 (1998) 505-518

[11] Margaret Symington, Generalized symplectic rational blowdowns, Algebr. Geom. Topol. 1 (2001) 503-518 (electronic)

[12] W P Thurston, HE Winkelnkemper, On the existence of contact forms, Proc. Amer. Math. Soc. 52 (1975) 345-347

[13] Ichiro Torisu, Convex contact structures and fibered links in 3-manifolds, Internat. Math. Res. Notices 2000 (2000) 441-454

[14] Bronislaw Wajnryb, An elementary approach to the mapping class group of a surface, Geom. Topol. 3 (1999) 405-466 (electronic)

[15] Alan Weinstein, Contact surgery and symplectic handlebodies, Hokkaido Math. J. 20 (1991) 241-251

Department of Mathematics, University of Arizona

617 North Santa Rita, PO Box 210089

Tucson, AZ 85721, USA

Email: dtgay@math.arizona.edu

Received: 27 January 2003 